\title{
Potentially inappropriate medicine prescribing by nurse practitioners in New Zealand
}

\author{
Betty Poot, RN, NP, BBS, MN (Nurse Practitioner, Lecturer)1,2, Katherine Nelson, RN, PhD (Senior Lecturer)' \\ Rebecca Zonneveld, RN, NP, MN (Nurse Practitioner, Lecturer)³, \& Mark Weatherall, BA, MBChB, MApplStats, \\ FRACP (Professor) ${ }^{4}$
}

\begin{abstract}
Background and purpose: Nurse practitioners (NPs) in New Zealand (NZ) prescribe a broad range of medicines; little is known about their prescribing practice for older adults. Potentially inappropriate medicines (PIMs) can cause more harm than benefit. This study reports on the prescribing of PIMs to older adults ( $\geq 65$ years) by NPs in NZ.

Methods: A subset analysis using data from NZ Ministry of Health pharmaceutical collection from 2013 to 2015 was completed. Data included NP registration number, medicines dispensed, patient age, sex, and NZ Deprivation level. Those $<65$ years were excluded. Beers 2015 criteria were used to identify the PIMs.

Results: There were 106 NPs that prescribed medicines to 12,410 patients aged $\geq 65$ years. One third of the patients were prescribed $\geq 1$ PIMss. Most (68.4\%) were prescribed one PIM; $21.9 \%$ two PIMs; $7.1 \%$ three PIMs; and $2.6 \%$ were prescribed $\geq 4$ PIMs. NPs prescribed an average of $14.9 \%$ PIMs. Primary care NPs were more likely to prescribe PIMs, compared to those with a scope of older adults and long-term conditions $(p \leq 0.001)$. The most common Beers 2015 PIMs prescribed were proton pump inhibitors, non-steroidal anti-inflammatory drugs, alpha blockers, hypnotics, tricyclic antidepressants, and benzodiazepines.

Implications for practice: NPs prescribe lower rates of PIMs to older adults than other prescribers in NZ. However, prescribing practices can be improved and the findings indicate that a more specific educational focus on prescribing to older adults is required. The findings provide an important baseline internationally for NP PIM prescribing and can be used by NPS, and educationally to review and improve practices.
\end{abstract}

Keywords: Beers criteria; nurse practitioners; older adult; potentially inappropriate medicines; prescribing.

Journal of the American Association of Nurse Practitioners 32 (2020) 220-228, ( 2019 American Association of Nurse Practitioners

DOI\# 10.1097/JXX.0000000000000239

\section{Introduction}

Nurse practitioners (NPs) in New Zealand (NZ) prescribe a broad range of medicines (Poot, Zonneveld, Nelson, \& Weatherall, 2017), but little is known about their prescribing practice for older people. Evaluating NPs prescribing for older adults is important as the NZ population is aging and older age affects drug absorption, metabolism, and elimination, contributing to increased susceptibility to adverse effects of medicines. In addition, older

${ }^{1}$ Graduate School of Nursing Midwifery and Health, Victoria University of Wellington, Newtown, Wellington, New Zealand, ${ }^{2}$ Hutt Valley District Hutt Board, Lower Hutt, Wellington, New Zealand, ${ }^{3}$ Evolve Youth Health, Wellington, New Zealand, ${ }^{4}$ Department of Medicine, University of Otago, Wellington, Newtown, Wellington, New Zealand Correspondence: Betty Poot, RN, NP, BBS, MN, Nurse Practitioner, Graduate School of Nursing Midwifery and Health, Victoria University of Wellington, Wellington, New Zealand. Tel: +64 4463 5363; Fax: +64 4 463 5442; E-mail: betty.poot@vuw.ac.nz

Received: 11 December 2018; revised: 26 March 2019; accepted 5 April 2019 adults have more long-term health conditions than younger adults and therefore, are more likely to be prescribed more medications. Some medicines can cause more harm than benefit to older adults (those $\geq 65$ years) and these 'Potentially Inappropriate Medicines' (PIMs) have been associated with adverse health outcomes, including falls and increased mortality (Jamieson et al., 2018; McMahon, Cahir, Kenny, \& Bennett, 2014; Muhlack, Hoppe, Weberpals, Brenner, \& Schottker, 2017). This study reports findings of NP prescribing related to PIMs dispensed to older adults from an analysis of routinely collected NZ Ministry of Health $(\mathrm{MOH})$ pharmaceutical collection data.

Internationally, several screening tools are available to identify PIMs. In clinical care, the most widely used criteria for safe prescribing in older adults are the American Geriatrics Society (AGS) Beers criteria (American Geriatrics Society Beers Criteria Update Expert, 2015). The goal of the Beers criteria is to improve the care of the older person by informing clinician choice of medicines with the aim of reducing exposure to PIMs. They have been 
developed specifically for use in primary and secondary healthcare, although excluding palliative and hospice care. The Beers criteria were first introduced in 1991 and were last updated in 2015. The 2015 updated list was developed after critical appraisal of the literature and evaluation by an expert panel with relevant clinical expertise and experience. Changes in the 2015 Beers criteria from previous versions include the recommendation to avoid antiarrhythmic drugs as first line treatment for atrial fibrillation, nitrofurantoin in those with reduced kidney function, and the avoidance of all non-benzodiazepines, benzodiazepines and hypnotics. Additionally, the revised criteria recommended that proton pump inhibitor use should be avoided without justification for longer than eight weeks of therapy. The 2015 Beers criteria are grouped into five categories: those medicines to avoid in all older adults, those to avoid in certain diseases or syndromes, those to be used with caution, those to be avoided or to be used with dose adjustment in people with reduced kidney functions, and those with drug-to-drug interactions (American Geriatrics Society Beers Criteria Update Expert, 2015).

\section{Potentially inappropriate medicine prescribing}

Several studies describe prescribing of PIMs to those aged $\geq 65$ years using the 1997, 2003, 2012, and 2015 Beers criteria (Grina \& Briedis, 2017; Lim et al., 2016; Morin, Fastbom, Laroche, \& Johnell, 2015; Ryan, O'Mahony, Kennedy, Weedle, \& Byrne, 2009). Findings from these studies report that between $18.3 \%$ and $47.2 \%$ of those $\geq 65$ years were prescribed at least one PIM. A systematic review by Tommelein et al. (2015), which included 52 studies on potentially inappropriate prescribing in community-dwelling older adults, reported an overall estimate of inappropriate prescribing of $22.6 \%$ (range $0-98 \%$ ). This wide range is likely due to the diversity of the screening tools used and different study designs. For those studies reporting the prevalence of PIM prescribing, the most frequent PIM medication groups included: benzodiazepines, hypnotics, sedatives, non-steroidal anti-inflammatory drugs (NSAIDs), and antidepressants (Grina \& Briedis, 2017; Lim et al., 2016; Morin et al., 2015; Ryan et al., 2009; San-Jose et al., 2015; Tommelein et al., 2015). Increasing age, female sex, increasing numbers of medications and co-morbidities, and living in an institution, were associated with an increased risk of being prescribed a PIM. In addition, Lim et al. (2016) in a retrospective survey of 25,810 outpatients aged 65 years or above reported that having five or more different prescribers was strongly associated with PIM prescription.

\section{Potentially inappropriate medicine prescribing in New Zealand}

A 2011 population-based study of the prevalence of PIM prescription in NZ reported that $40.9 \%$ of those aged 65 years or above were prescribed at least one PIM, and about half of this group were exposed to two or more PIMs. The most commonly dispensed PIMs included the medication group NSAIDs; and individually the medicines zopiclone and amitriptyline were prescribed (Narayan \& Nishtala, 2015). In an extension of an earlier NZ study, where researchers interviewed 320 community-dwelling people aged 75 years or above about their medicinetaking practices, similar results were reported (Nishtala, Bagge, Campbell, \& Tordoff, 2014; Tordoff, Bagge, Gray, Campbell, \& Norris, 2010). Nishtala et al. (2014) used the Beers 2012 criteria to assess PIM use and reported that $42.7 \%(n=135)$ of older adults were prescribed one PIM, and $10.3 \%(n=33)$ were prescribed two or more PIMs (range 0-5). Comparing this internationally, a study by Lim et al. (2016) reported that amongst a sample of 7,132 older adults, $27.6 \%$ were prescribed at least one PIM. Similarly to the other studies, NSAIDs (24\%), tricyclic antidepressants (16.8\%), and benzodiazepines (14.6\%) were the most common PIM groups to be prescribed in NZ. Also consistent with international research on PIM use, polypharmacy (San-Jose et al., 2015) and the prescription of psychotropic medication were associated with a higher rate of PIM exposure (Nishtala et al., 2014).

\section{New Zealand nurse practitioner background}

The NP role was established in NZ in 2001. NPs are registered by the Nursing Council of New Zealand (NCNZ) after completing a Nursing Masters qualification, prescribing practicum, and passing a portfolio and panel assessment. As part of the prescribing practicum, the nurse is mentored by an authorized prescriber (medical practitioner or NP) who works in the NPs' intended area of practice. Registration enables the NP to practice autonomously and prescribe medicines. Until June 2014, NPs could only prescribe from a limited list of medications; however, legislation changes to the Medicines Amendment Act 2013 gave NPs the same rights as medical prescribers, and NPs now prescribe medicines from the full NZ pharmaceutical schedule (Lim, North, \& Shaw, 2014; $N C N Z, 2018)$. While the number of NPs in NZ is low, there has been a steady increase in numbers; with the numbers registered doubling from 145 in 2015 to 326 in 2018 (NCNZ, 2018).

There are three published studies relating to NPs in NZ prescribing medicines (Gagan, Boyd, Wysocki, \& Williams, 2014; Pirret, 2012; Poot et al., 2017). Of relevance to this current study, Poot et al.'s (2017) 2-year analysis of dispensed prescription data from all NZ NPs $(n=129)$ showed that NPs prescribe a broad range of medications across all therapeutic groups. The most commonly prescribed medicines were antibiotics and analgesic medicines, and prescribing patterns were similar to other prescribers. Poot et al. also showed that NPs prescribe medicines across all age groups ranging from newborn to 103 years 
of age, of whom 12,410 were adults aged 65 years or above. The findings reported in this current paper examined PIMs prescribing to these 12,410 older patients.

\section{Aims}

The aims of this study were to:

1. Estimate the proportion of NPs who prescribe potentially inappropriate medication to older adults

2. Identify the most frequently prescribed potentially inappropriate medications under Beers 2015 criteria

3. Explore the association of NP area of practice and the prescribing of potentially inappropriate medications

4. Describe the characteristics of the older adult cohort.

Ethical approval for the study was received from the Human Ethics Committee, Victoria University of Wellington NZ (HEC 22502). All results are reported in aggregate so that individual NPs and patients cannot be identified.

\section{Methods}

This is a subset analysis of a retrospective observational study of the NZ MOH pharmaceutical collection database. This database records all the community or retailpharmacy claims for prescribed medicines that are listed in the NZ pharmaceutical schedule. The details of the data collection methods have been previously described (Poot et al., 2017) and so are only briefly summarized here.

The pharmaceutical collection was searched for a period of 24 months from July 2013 to June 2015 using the code "Nursing Council" and "Nurse Practitioner" as distinct identifiers. Data obtained included NP registration number, therapeutic information on all subsidized medicines dispensed. Patient data included: age, sex, and NZ Deprivation (NZDep) level. Patients aged $<65$ years were excluded from the analysis by applying a filter to the original dataset.

The Beers criteria (American Geriatrics Society Beers Criteria Update Expert, 2015) were used to identify the medications that were potentially inappropriate. As the $\mathrm{MOH}$ dataset did not have information on diseases or syndromes, we could only examine the medications presented in Table 2 of the 2015 Beers criteria. Each medicine in the Table $\mathbf{2}$ Beers criteria list was checked for the NZ equivalent medication, and where the therapeutic group was named, the NZ Pharmaceutical schedule was searched for all medicines available in that therapeutic group. Although aspirin and sliding scale insulin are included in the Beers 2015 criteria, both medications were excluded from the analysis, as drug dose information was unavailable in the dataset, and it was considered unlikely that community-pharmacy dispensed medications would include sliding scale insulin. Aspirin is only categorized as a PIM, if doses exceed $325 \mathrm{mg}$; and typically only low dose aspirin (<325 mg) is prescribed in NZ. The database was validated for errors, which mainly consisted of spelling, and data that were unlikely were also omitted, for example the two records where the patient's age was listed as greater than 110 years.

As NPs in NZ are no longer restricted to prescribing in pre-defined area of practice, all NPs who prescribed medicines to those aged $\geq 65$ years were included in the study independent of their area of practice. The NP registration number was used to identify the NPs' area of practice, and the patient's national health indicator number was used as the identifier for individual patients. The area of practice for each NP was determined using the information available on the Nursing Council of New Zealand (NCNZ) website. The NCNZ cluster NPs practice into six main areas: Addictions/ Mental Health, Emergency/Acute Care, Long Term Conditions/Specialties, Neonatal/Child/Youth, Older Adult, and Primary Care. Where NPs had more than one scope, they were assigned to their predominant scope. Following scope-of-practice classification, data were de-identified for analysis.

The NZDep provides deprivation information using a socio-economic score, where 1 represents the least deprived areas and 10 , the most deprived areas. The scores are calculated at meshblock level from population census data, using the following dimensions: household income, employment, qualifications, telecommunication availability, transport, care support, living space, and home ownership information (Salmond \& Crampton, 2012). The NZDep scores are useful for describing the association between socio-economic deprivation and health outcomes, and interventions such as prescribing. NZ Deprivation2013 is the most up-to-date version of the socio-economic score in NZ.

\section{Data analysis}

Simple data descriptions and tabulations of the NPS, patients and dispensed medications were used to describe the NPS and patients. The statistical analysis examined the ratio of the number of Beers 2015 PIMs per patient to the number of medications per patient, to estimate rates and relative rates of Beers 2015 PIMs by potential explanatory variables. In order to do this the number of Beers 2015 PIMs per patient was treated as a count variable and Poisson regression was used. To account for the total number of medications per patient, the logarithm of this was used as an offset in the Poisson regression, as is standard practice to estimate rates and relative rates. We were particularly interested in whether, within the group of older adults, there was an association between Beers 2015 prescription and older age. While age can be treated as a continuous variable in this setting, we felt, without loss of analysis information, that it would be 
useful to categorize patient ages into ranges by decade: 65 to $<75$ years, 75 to $<85$ years, and $\geq 85$ years, as this reflects a common clinical description of young-old, old, and oldest-old. SAS 9.4 statistical package was used for statistical analysis.

\section{Results \\ Nurse practitioners}

Of the 129 NPs who had written one or more prescriptions to 54,845 patients in the Poot et al. (2017) study, 106 (82\%) NPs prescribed medicines to 12,410 patients aged $\geq 65$ years. These 106 NPs prescribed a mean of 81.5 (SD \pm 75.3 ) medications (range 1-286). The average proportion of PIM medications prescribing by each NP was 14.9\% (Table 1). Nurse practitioners with addictions and mental health scope of practice had the highest average proportion of PIM prescribing (33.5\%); those with a primary care scope of practice had an average proportion of $15.4 \%$; those with a long-term conditions scope had an average proportion of $12.3 \%$; and those with an older adult scope of practice had the lowest average proportion of PIM prescribing of $11.1 \%$. Primary care NPs were more likely to prescribe PIMs, compared to those NPs with a scope of older adult and long-term conditions shown by a rate ratio of 1.26 (95\% confidence interval 1.14-1.40) for primary care scope and 1.69 (1.53-1.83) for long-term conditions, both $p<.001$. Four NPs with a child and youth health scope of practice had prescribed to older adults. While it appears that some NPs prescribed medicines outside of their main scope of practice, it should be noted that five NPs in the dataset had more than one scope of practice.

\section{Patients}

Table 2 shows the patient characteristics. The mean age of the patients was 75.6 (range 65-103), and 53.3\% were female. Patients predominantly identified as NZ European or other ethnicity (84.7\%) with $11 \%$ identifying as Māori (NZ indigenous people). Using the NZDep2013 score, $79 \%$ lived in the most socially deprived areas of NZ; NZDep2013 greater or equal to five.

The total number of medications prescribed was 52,849 in 12,410 patients; average of 4.3 (range 1-35) medications. For females this was 29,633 in 6,610 patients, mean (SD) 4.48 (4.30) and in males 23,216 in 5,800 patients, mean (SD) 4.00 (3.87). Using the Beers 2015 criteria, the average proportion of PIMs prescribed to individual patients was 10.8\% (Table 3 ) and this was $10.9 \%$ in females and $10.6 \%$ in males. This rate decreased with age from $12.1 \%$ for the $65-74$ years age range; 9.6\% for the $75-84$ years age range; and $9.0 \%$ for those aged $\geq 85$ years. Table 2 shows the overall number of PIMs prescribed across all patients. Of the 12,410 patients, a third (33.1\%, $n=4,111)$ were prescribed between one and seven PIMs. For females, this was 2,268/6,610 (34.3\%) and males $1843 / 5,800$ (31.8\%). For the different age ranges, the rate of at least one PIM was 2,217/6,356 (34.9\%) in those aged 65 to $<75$; $1,254 / 4,036$ (31.1\%) for 75 to <85; and 640/2018 (31.7\%) for those aged 85 and over. Of the total number of PIMs, most (68.4\%) were only prescribed one PIM; $21.9 \%$ were prescribed two PIMs; 7.1\% were prescribed three PIMs; and 2.6\% were prescribed four or more PIMs. One patient was prescribed seven PIMS.

\section{Medications}

Table 4 shows the 30 mostly commonly prescribed PIMs. Omeprazole was prescribed to 1,641 (13.2\%) patients; Zopiclone to 474 (3.8\%); Ibuprofen to 440 (3.5\%); Doxazosin to 408 (3.3\%); Pantoprozole to 262 (2.1\%); and Amitriptyline to 250 (2\%) patients. In descending order, the most common Beers 2015 PIM therapeutic group dispensed were proton pump inhibitors 15.7\% $(n=1953)$, NSAIDs 7\% ( $n=872)$, alpha blockers 4.3\% ( $n=532)$, hypnotics 3.8\% ( $n=474)$, tricyclic antidepressants 3.3\% ( $n=$ 411), and benzodiazepines $2.9 \%(n=370)$.

Of the 106 prescribing NPs, 76 (70.8\%) prescribed Omeprazole, 63 (59.4\%) prescribed Zopiclone, 59 (55.7\%) prescribed Ibuprofen, 55 (51.9\%) Doxazosin, and 45 (42.5\%) prescribed Amitriptyline at least once.

\section{Discussion}

Proton pump inhibitors, NSAIDs, alpha blockers, hypnotics, tricyclic antidepressants, and benzodiazepines were the most commonly prescribed PIM groups by NPS. With the exception of the proton pump inhibitor therapeutic group, this finding is consistent with previous NZ and international studies (Grina \& Briedis, 2017; Lim et al., 2016; Morin et al., 2015; Narayan \& Nishtala, 2015; Nishtala et al., 2014; Ryan et al., 2009; San-Jose et al., 2015; Tommelein et al., 2015). The finding concerning proton pump inhibitors is likely to be inconsistent because earlier versions of the Beers criteria did not include these as a PIM and as a result, previous studies did not report their use. This therapeutic group was added to Beers in the 2015 criteria as there was strong evidence to indicate an association between proton pump inhibitors and clostridium difficile infections, bone loss, and fractures. Although the Beers 2015 criteria were published after we obtained the dataset, the evidence that prolonged exposure (>8 weeks) to proton pump inhibitors is contraindicated for older adults was available for prescribing clinicians prior to 2015 (American Geriatrics Society Beers Criteria Update Expert, 2015; Maes, Fixen, \& Linnebur, 2017).

The high prevalence of prescriptions by NPs for amitriptyline and zopiclone is of concern. These medications are contraindicated in older persons, as they contribute to a greater number of adverse effects. These effects were recently highlighted in a NZ study by Jamieson (2018) which showed there was a higher incidence of falls in the 
Table 1. Number and proportion of Beers 2015 potentially inappropriate medications prescribed at least once to a patient aged $\geq 65$ years by nurse practitioner scope of practice

\begin{tabular}{|c|c|c|c|c|c|c|c|}
\hline & $\begin{array}{l}\text { All NPs } \\
N=106\end{array}$ & $\begin{array}{l}\text { Addictions and } \\
\text { Mental Health } \\
\qquad N=7\end{array}$ & $\begin{array}{c}\text { Emergency and } \\
\text { Acute Care } \\
N=7\end{array}$ & $\begin{array}{l}\text { Long Term } \\
\text { Conditions } \\
\qquad N=29\end{array}$ & $\begin{array}{l}\text { Neonatal Child } \\
\text { and Youth } \\
N=4\end{array}$ & $\begin{array}{l}\text { Older } \\
\text { Adult } \\
N=14\end{array}$ & $\begin{array}{c}\text { Primary } \\
\text { Care } \\
\mathbf{N}=\mathbf{4 5}\end{array}$ \\
\hline \multicolumn{8}{|l|}{ No. of PIMs } \\
\hline Mean (SD) & $10.5(10.1)$ & $4.0(4.2)$ & $3.1(2.4)$ & $5.8(5.0)$ & $0.8(1.5)$ & $7.9(7.7)$ & $17.4(10.7)$ \\
\hline Median (IQR) & $7.5(3-16)$ & $2(1-9)$ & $3(1-5)$ & $4(2-8)$ & $0(0-1.5)$ & $4.5(3-16)$ & $16(10-26)$ \\
\hline Min to $\max$ & $0-43$ & $1-11$ & $0-7$ & $0-23$ & $0-3$ & $0-24$ & $0-43$ \\
\hline \multicolumn{8}{|c|}{ No. of total medications } \\
\hline Mean (SD) & $81.5(75.3)$ & $16.3(19.9)$ & $28.9(24.9)$ & $47.7(36.3)$ & $2.3(1.9)$ & $66(56.6)$ & $133.5(79.6)$ \\
\hline Median (IQR) & $56(26-120)$ & $8(3-32)$ & $28(7-39)$ & $39(29-55)$ & $1.5(1-3.5)$ & $45.5(16-113)$ & $120(79-203)$ \\
\hline Min to $\max$ & $1-286$ & $2-55$ & $6-77$ & $1-159$ & $1-5$ & 8-188 & $1-286$ \\
\hline \multicolumn{8}{|c|}{ aproportion of PIMS (\%) } \\
\hline Mean (SD) & $14.9(13.5)$ & $33.5(17.8)$ & $11.2(5.9)$ & $12.3(9.6)$ & $15.0(30.0)$ & $11.1(6.2)$ & $15.4(13.7)$ \\
\hline Median (IQR) & $12.9(10.1-15.7)$ & $25.0(20.0-50.0)$ & $10.7(9.1-16.7)$ & $10.7(6.9-14.8)$ & $0(0-30)$ & $12.6(8.2-14.2)$ & $13.5(11.5-14.9)$ \\
\hline Min to $\max$ & $0-100$ & $20.0-66.7$ & $0-16.7$ & $0-50$ & $0-60$ & $0-23.1$ & $0-100$ \\
\hline
\end{tabular}

Note: $I Q R$ = interquartile range; $N P=$ Nurse practitioner; $P I M=$ potentially inappropriate medicine

${ }^{a}$ The proportion of PIMs is the number of patient-Beers PIM combinations divided by the number of total patient-medication combinations. 


\section{Table 2. Patient characteristics}

\begin{tabular}{|c|c|c|c|c|}
\hline Variable & Results & Mean (SD) & Median (IQR) & Range \\
\hline Age (years) & & $75.6(8.0)$ & $74(69-81)$ & $65-103$ \\
\hline \multicolumn{5}{|l|}{$N=12,408^{a}$} \\
\hline NZ deprivation index 2013 & & $6.9(2.6)$ & $7(5-9)$ & $1-10$ \\
\hline \multicolumn{5}{|l|}{$N=12,240^{b}$} \\
\hline & N/12410 (\%) & & & \\
\hline Sex (female) & $6,610(53.3)$ & & & \\
\hline \multicolumn{5}{|l|}{ Ethnicity } \\
\hline Asian & $214(1.7)$ & & & \\
\hline European or other & $10,511(84.7)$ & & & \\
\hline Māori & $1,368(11.0)$ & & & \\
\hline Pacific & $317(2.6)$ & & & \\
\hline \multicolumn{5}{|l|}{ No. of PIMs per patient } \\
\hline 0 & $8,299(66.9)$ & & & \\
\hline 1 & $2,810(22.6)$ & & & \\
\hline 2 & $900(7.3)$ & & & \\
\hline 3 & $293(2.4)$ & & & \\
\hline 4 & $86(0.7)$ & & & \\
\hline 5 & $16(0.1)$ & & & \\
\hline 6 & $5(0.04)$ & & & \\
\hline 7 & $1(0.01)$ & & & \\
\hline
\end{tabular}

Note: $I Q R$ = interquartile range; $P I M=$ potentially inappropriate medicine.

${ }^{a}$ Two records with age 110 or greater.

${ }^{b}$ Missing data for $\mathrm{N}=170$.

elderly who had a higher drug burden index, which is related to prescription of medications with anticholinergic properties or sedative medicines. As falls are associated with increased morbidity and mortality, these medications should be avoided in older persons.

This study shows that the majority of NPs in NZ $(106 / 129,82 \%)$ prescribe medicines to those aged 65 years and over. Twenty-three NPs did not prescribe medicines to this age group and the majority of these had a scope of practice limited to neonatal, child and youth care. While the average proportion of PIM prescribing by NPS to the older adult is relatively low at $14.9 \%$, the patient exposure to one or more PIM was 33.1\%. Although this patient exposure rate is lower than the exposure rate reported in the two other NZ studies (Narayan \& Nishtala, 2015; Nishtala et al., 2014) which reported rates of $40.9 \%$ and $42.7 \%$, the rate is higher than reported in some in-

ternational studies (Grina \& Briedis, 2017; Lim et al., 2016; Morin et al., 2015; Ryan et al., 2009).
The finding that NPs working in primary care prescribed more PIMs than those who had a long-term conditions or older person's scope of practice is interesting. Explanations for this are likely to be educational and practice-based. Educational factors may include content of Masters education, types of professional development, and role of mentors, but the nature of the dataset meant that these factors could not be explored. As placement during NP training involves individual mentors, the NPS will have had different experiences and opportunities. The nature of NP work is such that, different NPs work with different patient populations. NPs in primary care, work across different age groups and may therefore be less aware than NPs who work solely with older persons, that specific criterion such as Beers should be used for guidance, when prescribing to older adults. It is not surprising that those NPs solely working under an addictions and mental health scope of practice have the highest proportion of PIM prescribing, 
Table 3. Number and proportion of Beers 2015 potentially inappropriate medications prescribed to individual patients aged 65 years and over by age

\section{All Aged $\geq 65$ Years $N=12,410$}

No. of PIMS

\begin{tabular}{|c|c|c|c|c|}
\hline Mean (SD) & $0.48(0.81)$ & $0.49(0.80)$ & $0.45(0.80)$ & $0.49(0.87)$ \\
\hline Median (IQR) & $0(0-1)$ & $0(0-1)$ & $0(0-1)$ & $0(0-1)$ \\
\hline Min to $\max$ & $0-7$ & $0-6$ & $0-7$ & $0-6$ \\
\hline \multicolumn{5}{|c|}{ No. of total medications } \\
\hline Mean (SD) & $4.3(4.1)$ & $4.2(3.8)$ & $4.2(4.2)$ & $4.5(4.7)$ \\
\hline Median (IQR) & $3(1-6)$ & $3(1-6)$ & $3(1-6)$ & $2(1-7)$ \\
\hline Min to $\max$ & $1-35$ & $1-27$ & $1-35$ & $1-33$ \\
\hline \multicolumn{5}{|c|}{ Proportion of PIMs (\%) } \\
\hline Mean (SD) & $10.8(21.9)$ & $12.1(23.5)$ & $9.6(20.5)$ & $9.0(18.9)$ \\
\hline Median (IQR) & $0(0-14.3)$ & $0(0-16.7)$ & $0(0-12.1)$ & $0(0-11.8)$ \\
\hline Min to $\max$ & $0-100$ & $0-100$ & $0-100$ & $0-100$ \\
\hline
\end{tabular}

Note: $I Q R=$ interquartile range; $P I M=$ potentially inappropriate medicine.

as medications used in the treatment of these conditions often have anticholinergic or sedative effects.

The results indicate that more education for all NPS focusing on prescribing to older adults is required. Educators and mentors providing support for intern NPs, independent of intended scope of practice, should include more in-depth and focused education on prescribing to older adults as a core component during the prescribing practicum. Tertiary education institutions providing NP programs need to develop professional development opportunities and resources to enhance NP prescribing practices, particularly for those working in primary care and mental health. In addition, NPs should complete regular medicine reviews with their patients and with colleagues to reassess the appropriateness of any prescribed medication. NPs, either alone or as part of peer review, should consider auditing their own prescribing practice against such criteria as the Beers criteria as a way to critically examine and enhance their prescribing practice.

This study is a part of a growing body of evidence of NP prescribing in the NZ healthcare setting. Further research evaluating the impact of the role of mentorship during NP practicum placements, and the effectiveness of targeted education on prescribing patterns is necessary in NZ and internationally to ensure NPs maintain appropriate and safe prescribing practices. In addition, evaluating patterns of prescribing across socio-economic and ethnic groups will be important to establish if any groups are disadvantaged by NP prescribing.

\section{Limitations and strengths}

This study is a subset of a larger study examining the types and rates of medicines prescribed by NPs in NZ. A major strength of the dataset is that it is a complete dataset of NP dispensed prescriptions in NZ. Using the dataset rather than recalling what medicines have been prescribed means that the data of dispensed prescription are relatively strong. However, the dataset only contains information of dispensed medications that are subsidized by the NZ government; therefore, those medications that are not subsidized; those prescriptions written but not filled by the patient; or those medicines purchased over the counter at a community pharmacy are not included in this analysis. From the dataset it is impossible to determine how many patients were in palliative or hospice care; however, only four NPs have a scope of practice in adult palliative care so the effect of this would be relatively minor. As the NZ pharmaceutical schedule does not contain all the medications that are listed in the Beers 2015 criteria, this study is limited to those medications that are publicly available in NZ. In addition, information on health conditions, renal function, the initial start date for the medication, dose of medication, and whether the medication dose was being down titrated was unavailable. Due to this, we did not examine the medications that should be avoided in drug to disease, drug to syndrome categories and those that should be avoided in patients with decreased renal functions. 
Table 4. Frequency of the $\mathbf{3 0}$ most prescribed

Beers 2015 potentially inappropriate

medications by nurse practitioner and number of patients

\begin{tabular}{|c|c|c|}
\hline Chemical Name & $\begin{array}{c}\text { N/106 } \\
\text { NPs (\%) }\end{array}$ & $\begin{array}{c}N / 12410 \\
\text { Patients (\%) }\end{array}$ \\
\hline Omeprazole & $75(70.8)$ & $1641(13.2)$ \\
\hline Zopiclone & $63(59.4)$ & $474(3.8)$ \\
\hline Ibuprofen & $59(55.7)$ & $440(3.5)$ \\
\hline Doxazosin & $55(51.9)$ & $408(3.3)$ \\
\hline Pantoprazole & $50(47.2)$ & $262(2.1)$ \\
\hline Diclofenac sodium & $48(45.3)$ & $209(1.7)$ \\
\hline Oestriol & $46(43.4)$ & $221(1.8)$ \\
\hline Amitriptyline & $45(42.5)$ & $250(2.0)$ \\
\hline Digoxin & $42(39.6)$ & $227(1.8)$ \\
\hline Nitrofurantoin & $39(36.8)$ & $197(1.6)$ \\
\hline Naproxen & $38(35.8)$ & $192(1.5)$ \\
\hline Terazosin & $36(34.0)$ & $119(1.0)$ \\
\hline Lorazepam & $35(33.0)$ & $135(1.1)$ \\
\hline Nortriptyline hydrochloride & $34(32.1)$ & $111(0.9)$ \\
\hline Amiodarone hydrochloride & $32(30.2)$ & $69(0.6)$ \\
\hline Dipyridamole & $30(28.3)$ & $85(0.7)$ \\
\hline Metoclopramide hydrochloride & $29(27.4)$ & $143(1.2)$ \\
\hline Paroxetine hydrochloride & $29(27.4)$ & $80(0.6)$ \\
\hline Clonazepam & $28(26.4)$ & $86(0.7)$ \\
\hline Diazepam & $28(26.4)$ & $44(0.4)$ \\
\hline Hyoscine hydrobromide & $26(24.5)$ & $16(0.1)$ \\
\hline Lansoprazole & $22(20.8)$ & $50(0.4)$ \\
\hline Triazolam & $20(18.9)$ & $49(0.4)$ \\
\hline Orphenadrine citrate & $20(18.9)$ & $46(0.4)$ \\
\hline Promethazine hydrochloride & $20(18.9)$ & $30(0.2)$ \\
\hline Temazepam & 19 (17.9) & $45(0.4)$ \\
\hline Doxepin hydrochloride & 19 (17.9) & $40(0.3)$ \\
\hline Nifedipine & $12(11.3)$ & $21(0.2)$ \\
\hline Oxazepam & $11(10.4)$ & $15(0.1)$ \\
\hline Clonidine hydrochloride & $10(9.4)$ & $14(0.1)$ \\
\hline
\end{tabular}

Note: $N P=$ Nurse practitioner

\section{Implications and relevance of this study}

We believe this is the first study to examine NP prescribing practices in the older people. It has identified the PIMs that NPs prescribe to older adults. This knowledge is important for preparing NP interns to prescribe, as well as for providing already registered NPs with important topics for ongoing professional development. The findings also contribute to the growing body of knowledge on NP prescribing, in general.

These results provide further evidence that the standard of NPs prescribing practice to older people is positively comparable to other prescribers and is further a confirmation that NPs are well prepared to prescribe. While the rates of PIM prescribing are similar to other prescribers, any reduction in the prescribing of PIMs has the potential to improve outcomes for older adults. Knowledge of NP prescribing practices, in particular, can help to guide those tertiary institutions that provide NP training programs to develop course content that is relevant to improving pharmacotherapy in older persons. In addition, this should be the focus of ongoing professional development programs for all NPs. This is particularly relevant for NZ NPs, as from 2017 NCNZ removed the scope of practice conditions for all newly registered NPs and all currently registered NPs can apply to have their scope of practice conditions removed (NCNZ 2018).

The findings of this study are timely and can be used to inform curricula development for NP prescribing practicum and continuing education courses. This knowledge could also be used to add to the content of registered nurse prescribing practicum, as they too can prescribe medicines (albeit from a more limited list of medications) for older adults.

\section{Conclusion}

This study provides an important baseline for understanding NP prescribing of PIMs in NZ and internationally. The majority of NPs practicing in NZ prescribe medications to older adults. The average proportion of PIM prescribing by each NP appears relatively low; yet the patient exposure to one or more PIM is high. Proton pump inhibitors, NSAIDs, alpha blockers, hypnotics, tricyclic antidepressants, and benzodiazepines were the most commonly prescribed PIM by NPs. Their PIM prescribing patterns are similar to other prescribers nationally and internationally, except for the proton pump inhibitor therapeutic group. It is difficult to compare this therapeutic group across all prescribers as it was not included in previous versions of the AGS Beers criteria.

This study has relevance internationally, given the increasingly aging population. Nurse practitioners can use the findings of this study as starting point to reflect on their own prescribing practice. Understanding NP prescribing patterns for older adults, is important to ensure educational content of NP prescribing practicums and professional development focuses on appropriate and safe prescribing. 
Acknowledgments: The authors would like to acknowledge Emmanuel Jo from Health Workforce New Zealand who completed the data extraction for this study.

Authors' contributions: $B$. Poot wrote the initial draft of the manuscript; $M$. Weatherall completed the statistical analysis; all authors contributed to the paper and revised the manuscript for final submission.

\section{Competing interests: The authors report no conflicts of} interest.

\section{References}

American Geriatrics Society Beers Criteria Update Expert, P. (2015). American Geriatrics Society 2015 updated Beers criteria for potentially inappropriate medication use in older adults. Journal of the American Geriatrics Society, 63, 2227-2246.

Gagan, M. J., Boyd, M., Wysocki, K., \& Williams, D. J. (2014). The first decade of nurse practitioners in New Zealand: A survey of an evolving practice. Journal of the American Association of Nurse Practitioners, 26, 612-619.

Grina, D., \& Briedis, V. (2017). The use of potentially inappropriate medications among the Lithuanian elderly according to Beers and EU(7)-PIM list - a nationwide cross-sectional study on reimbursement claims data. Journal of Clinical Pharmacy and Therapeutics, 42, 195-200.

Jamieson, H., Nishtala, P. S., Scrase, R., Deely, J. M., Abey-Nesbit, R., Hilmer, S. N.,... Schluter, P. J.. (2018). Drug burden index and its association with hip fracture among older adults: A national population-based study. The Journals of Gerontology: Series A. doi:10.1093/gerona/gly176

Lim, A. G., North, N., Shaw, J. (2014). Nurse prescribing: The New Zealand context. Nursing Praxis in New Zealand, 30, 18-27.

Lim, Y. J., Kim, H. Y., Choi, J., Lee, J. S., Ahn, A. L., Oh, E. J., ... Kweon, H. J. (2016). Potentially inappropriate medications by Beers Criteria in older outpatients: Prevalence and risk factors. Korean Journal of Family Medicine, 37, 329-333.

Maes, M. L., Fixen, D. R., \& Linnebur, S. A. (2017). Adverse effects of proton-pump inhibitor use in older adults: A review of the evidence. Therapeutic Advances in Drug Safety, 8, 273-297.

McMahon, C. G., Cahir, C. A., Kenny, R. A., \& Bennett, K. (2014). Inappropriate prescribing in older fallers presenting to an Irish emergency department. Age and Ageing, 43, 44-50.

Morin, L., Fastbom, J., Laroche, M. L., \& Johnell, K. (2015). Potentially inappropriate drug use in older people: A nationwide comparison of different explicit criteria for population-based estimates. British Journal of Clinical Pharmacology, 80, 315-324.

Muhlack, D. C., Hoppe, L. K., Weberpals, J., Brenner, H., \& Schottker, B. (2017). The association of potentially inappropriate medication at older age with cardiovascular events and overall mortality: A systematic review and meta-analysis of cohort studies. Journal of the American Medical Directors Association, 18, 211-220.

Narayan, S. W., \& Nishtala, P. S. (2015). Prevalence of potentially inappropriate medicine use in older New Zealanders: A populationlevel study using the updated 2012 Beers criteria. Journal of Evaluation in Clinical Practice, 21, 633-641.

NCNZ. (2018). Nurse Practitioner. Retrieved from http://www.nursingcouncil.org.nz/Nurses/Scopes-of-practice/Nursepractitioner.

Nishtala, P. S., Bagge, M. L., Campbell, A. J., \& Tordoff, J. M. (2014). Potentially inappropriate medicines in a cohort of communitydwelling older people in New Zealand. Geriatrics Gerontology International, 14, 89-93.

Pirret, A. M. (2012). A critical care nurse practitioner's prescribing using standing orders and authorised prescribing when performing a critical care outreach role: A clinical audit. Intensive \& Critrical Care Nursing, 28, 1-5.

Poot, B., Zonneveld, R., Nelson, K., \& Weatherall, M. (2017). Prescribing by nurse practitioners: Insights from a New Zealand study. Journal of the American Association of Nurse Practitioners, 29, 581-590.

Ryan, C., O'Mahony, D., Kennedy, J., Weedle, P., \& Byrne, S. (2009). Potentially inappropriate prescribing in an Irish elderly population in primary care. British Journal of Clinical Pharmacology, 68, 936-947.

Salmond, C. E., \& Crampton, P. (2012). Development of New Zealand's deprivation index (NZDep) and its uptake as a national policy tool. Canadian Journal of Public Health, 103(8 suppl 2), S7-S11.

San-Jose, A., Agust, A., Vidal, X., Formiga, F., Gomez-Hernandez, M., Garcia, J., ... Barbé, J. (2015). Potentially Inappropriate Prescription in Older Patients in Spain (PIPOPS) Investigators' project. Inappropriate prescribing to the oldest old patients admitted to hospital: Prevalence, most frequently used medicines, and associated factors. BMC Geriatrics, 15, 42.

Tommelein, E., Mehuys, E., Petrovic, M., Somers, A., Colin, P., \& Boussery, K. (2015). Potentially inappropriate prescribing in community-dwelling older people across Europe: A systematic literature review. European Journal of Clinical Pharmacology, 71, 1415-1427.

Tord off, J. M., Bagge, M. L., Gray, A. R., Campbell, A. J., \& Norris, P. T. (2010). Medicine-taking practices in community-dwelling people aged $>=75$ years in New Zealand. Age and Ageing, 39, 574-580. 